DOI: https://doi.org/10.11144/Javeriana.upsy20.bfep

\title{
Barreras y factores de éxito percibidos por el profesorado universitario. Diferencias por género y categorías académicas*
}

\section{Perceived Barriers and Success Factors by University Faculty. Differences by Gender and Academic Ranks}

\author{
Nazareth Gallego-Morón ${ }^{\mathrm{a}}$ \\ Universidad Pablo de Olavide, España \\ ORCID: https://orcid.org/0000-0002-8172-4515 \\ Mauricio Matus-López \\ Universidad Pablo de Olavide, España \\ ORCID: https://orcid.org/0000-0002-5273-823X \\ Lina Gálvez Muñoz \\ Universidad Pablo de Olavide, España \\ ORCID: https://orcid.org/0000-0002-9645-2778
}

a Autor de correspondencia. Correo electrónico: ngalmor1@upo.es

Para citar este artículo: Gallego-Morón, N., MatusLópez, M., \& Gálvez Muñoz, L. (2021). Barreras y factores de éxito percibidos por el profesorado universitario. Diferencias por género y categorías académicas. Universitas Psychologica, 20, 1-18. https://doi.org/10.11144/Javeriana.upsy20.bfep

\section{RESUMEN}

Este artículo explora en qué medida los factores personales, organizacionales y sociales determinan las trayectorias del profesorado universitario, controlando variables sociodemográficas como sexo y rango académico. Para ello, se desarrolló un estudio de tipo correlacional, en una muestra de profesorado de la Universidad Federico II de Nápoles, en Italia ( $N=228,55.3 \%$ hombres y $44.7 \%$ mujeres), de entre 21 y 67 años $(M=51.7, D E=9.2)$, con el empleo de estadística descriptiva y análisis de correspondencia múltiple. Los hallazgos evidencian dos tendencias diferenciadas: (1) la del profesorado masculino y de mayor nivel y (2) la de las mujeres y el profesorado de categorías medias e inferiores. Los primeros perciben menos dificultades y valoran más los factores de éxito personales (Modelo 1, 53.9\% de la varianza) y familiares (Modelo 2, 50.3 $\%$ de la varianza). Barreras como la influencia de redes informales de poder (Modelo 3, $42.7 \%$ de la varianza) y cuestiones familiares, como la dificultad de conciliación familiar, personal y laboral (Modelos 4 y 5, 50.2 \% y $59.9 \%$ de la varianza), son percibidos con mayor intensidad por el segundo grupo. A raíz de estos datos, se destaca la importancia de medidas en pro de la igualdad de oportunidades en la academia.

\section{Palabras clave}

barreras; carrera investigadora; género; profesorado universitario; factores de éxito.

\begin{abstract}
This paper explores to what extent individual, organizational and social factors determine academic trajectories, controlling for sociodemographic variables such as sex and academic rank. For this, a correlational study was developed in a convenience sample of academics from the Federico II University of Naples, in Italy $(N=228,55.3 \%$ men and $44.7 \%$ women), between 21 and 67 years old $(M=51.7, S D=9.2)$, with the use of descriptive statistics and multiple correspondence analysis. The results show two differentiated tendencies: (1) that of male and full-
\end{abstract}


professors, and (2) that of women and middle and earlycareer levels. The first group perceive less difficulties and value personal (Model 1, $53.9 \%$ of variance) and family success (Model 2, $50.3 \%$ of variance) factors more. Barriers such as the influence of informal power networks (Model 3, 42.7 \% of variance) and family issues, as work-life balance (Model 4 and 5, 50.2 \% and 59.9\% of variance), are perceived with greater intensity by the second group. The results highlight the importance of measures for equal opportunities in academia.

Keywords

barriers; research career; gender; faculty; universities; success factors.

La UNESCO describe la educación superior como feminizada (Instituto de Estadística de la UNESCO [UIS], 2018). En el $80 \%$ de países con datos disponibles, la mayor parte del alumnado son mujeres, así como en las tasas de egreso de grado y de máster. No obstante, en los estudios de doctorado bajan de la mitad, y en el personal investigador son solo tres de cada diez (UIS, 2020). Esta diferencia aumenta en las categorías más altas y en los cargos de gestión (European Commission, 2019). En la actualidad, poco más de un $10 \%$ de las universidades italianas están dirigidas por mujeres (Conferenza dei Rettori delle Università italiane [CRUI], 2020) y en el puesto más alto, el de profesorado ordinario, representaban el $24 \%$, en el curso 2018/19 (Ministero dell'Istruzione dell'Università e della Ricerca [MIUR], 2020).

Este fenómeno se conoce como techo de cristal y se refiere a la existencia de una barrera invisible $\mathrm{y}$ artificial que dificulta a las mujeres promocionar a la cúspide de las organizaciones (Morrison et al., 1987, p. 13). La concepción original ha evolucionado, y más que describirse como una única pared sólida, parece ajustarse mejor a una especie de laberinto(s) de cristal (Picardi, 2017). Es decir, un entramado de obstáculos invisibles presentes desde el inicio de la carrera académica y que se acentúa al final de esta (Reynolds et al., 2018). Diversos estudios internacionales (p. ej., Bonache et al., 2020; Corroy \& Savignac, 2016; Franco-Orozco \& Franco-Orozco, 2018; Rebolledo \& Espinoza, 2016; Thun, 2020; Van der Lee \& Ellemers, 2015; Wesarat \& Mathew, 2017) realizados en países como España, Francia,
Colombia, Chile, Noruega, Países Bajos o India han identificado esos obstáculos. Sin embargo, no existe consenso acerca de cuáles son ni sobre su tipología (Gallego-Morón \& Matus-López, 2018; Gallego-Morón et al., 2020).

Frente a la proliferación de investigaciones centradas en las causas del fenómeno, es escasa la literatura que se ha ocupado de investigar cuáles son los factores que han propiciado el éxito de las académicas (Collings et al., 2011; Fritsch, 2015; Gallego-Morón \& Matus-López, 2020). Aquellos que sí lo han hecho se centran en el análisis de las experiencias de mujeres en cargos universitarios relevantes (Saleem \& Ajmal, 2018). No obstante, pocos presentan como objeto de estudio el conocimiento de los apoyos o estrategias adoptadas por las mujeres que se encuentran en las primeras posiciones universitarias (Reynolds et al., 2018).

Además, es limitada la literatura que analiza la relación entre barreras y factores de éxito, así como las diferencias por género y categoría académica. Para el caso de Italia no consta la existencia de investigaciones previas que, considerando la estructuralidad del fenómeno, estudien los aspectos positivos y negativos que el profesorado encuentra en las diferentes etapas de la carrera. Por todo ello, el objetivo del presente trabajo consiste en contrastar hasta qué punto factores personales, organizacionales y sociales condicionan las trayectorias universitarias, y si existe una relación entre la valoración de estas y las variables sexo y rango ocupado.

De este modo, las variables de estudio contempladas fueron: el sexo (Hombre/Mujer), la categoría académica (profesorado ordinario, associato y ricercatore), las barreras (numeradas b1 a b14) y los factores de éxito (numerados e1 a e16), entendidos como aquellos condicionantes que suponen una dificultad o un impulso positivo, respectivamente, en el normal desarrollo de la labor profesional, y por ende, en las posibilidades de promoción. Conocer los factores que afectan tanto al fracaso como al éxito del profesorado, en general, y de las mujeres, en particular, ayuda a la comunidad científica y a los organismos públicos, otorgando un punto de partida fundamental para el diseño y aplicación de medidas que 
promuevan la igualdad de oportunidades en la carrera científica e investigadora.

\section{Antecedentes bibliográficos}

El laberinto de cristal (o laberintos de cristal) se constituye como un fenómeno estructural, determinado por una amplia variedad de condicionantes de diversa tipología (Collings et al., 2011). Factores que favorecen el éxito en la carrera académica y barreras que impiden avanzar en ella. Son dos caras de una misma moneda, pero que afectan de forma distinta a las personas. En el presente trabajo, se ha realizado una revisión exhaustiva de la bibliografía científica, lo que ha permitido clasificar estos factores y barreras en tres tipos: condicionantes personales (cognitivo-emocionales y familiares), organizacionales (grupales y profesionales) y sociales. A continuación, se describen cada uno de ellos.

\section{Condicionantes personales cognitivo- emocionales}

Aspectos como la motivación, la pasión por el trabajo y la vocación, junto con el esfuerzo y la dedicación, son señalados por la literatura como condicionantes positivos que facilitan el desempeño de la labor científica e investigadora (Gallego-Morón \& Matus-López, 2020; Reynolds et al., 2018). En esta línea, resultados de investigaciones anteriores como las de Redmon et al. (2017) a académicas de Australia en cargos de gestión relevantes coinciden con los encontrados por Collings et al. (2011) para el mismo país, o los de Franco-Orozco y Franco-Orozco (2018) para Colombia. Estos señalan como factores de éxito la combinación entre la adopción de ciertas actitudes y características personales. Comportamientos como la proactividad, la perseverancia y la capacidad para tomar riesgos y decisiones en el trabajo, acompañados de autoconfianza y un fuerte carácter para saber encajar posibles fracasos y responder a las dificultades existentes, desarrollando altos niveles de resiliencia, se presentan como aspectos fundamentales para la promoción en la carrera.

Sin embargo, una parte de la literatura señala la existencia de barreras cognitivo-emocionales que obstaculizan la promoción femenina, como un bajo nivel de autoestima (Collings et al., 2011; Vayreda et al., 2019), falta de confianza en sí mismas o miedo ante la inseguridad laboral (Cooper, 2019). Bozzon et al. (2017) analizan el impacto de la maternidad/paternidad en las carreras del personal investigador de una universidad italiana. Muestran que las mujeres con hijos/as en ocasiones presentan sentimientos de culpabilidad ante las dificultades para conciliar la esfera familiar y laboral, no prestando la atención que desearían a esta última. Como consecuencia, estas sienten un conflicto interno (Bonache et al., 2020; Reynolds et al., 2018), lo que influye en su autoexclusión, en mayor medida que los varones, quienes tomarían la decisión personal de no mejorar su puesto académico, priorizando la vida familiar (Gorshkova \& Miryasova, 2020; Rebolledo \& Espinoza, 2016; Thun, 2020).

\section{Condicionantes personales familiares}

La evidencia empírica señala la influencia tanto positiva como negativa de este tipo de factores. La asunción de responsabilidades derivadas de las tareas domésticas y de cuidados, y las dificultades para conciliar estas con la esfera laboral, se constituyen como una de las principales barreras que las mujeres afrontan en sus carreras (Bonache et al., 2020; Castañeda-Rentería et al., 2019; Gallego-Morón et al., 2020). A pesar de que el número de mujeres trabajadoras ha aumentado, siendo cada vez más comunes las familias donde ambos miembros trabajan, sigue considerándose en el imaginario colectivo que son las mujeres quienes deben asumir las tareas no remuneradas (Gorshkova \& Miryasova, 2020; Toffoletti \& Starr, 2016).

Todo ello repercute en la disponibilidad de tiempo, dedicación, movilidad, etc., afectando sus niveles de estrés y ansiedad, así como, en definitiva, la capacidad para trabajar y como 
consecuencia, las posibilidades de promoción (Gallego-Morón \& Matus-López, 2020; Obiol \& Villar, 2016). Esta situación se ve potenciada en el caso de las primeras etapas académicas, coincidiendo con la edad reproductiva. Como demuestran Bozzon et al. (2017) en la investigación realizada al personal investigador postdoctoral italiano, en ocasiones, las mujeres deciden abandonar sus carreras o reducir la intensidad productiva. En el caso de España, Bonache et al. (2020) encontraron que las académicas de las primeras etapas percibían más barreras después de tener hijos/as, en comparación con los varones. Estas ocupan más tiempo y presentan niveles más altos de preocupación hacia la conciliación profesional y familiar. Esta situación afecta en menor medida las posibilidades de éxito de los hombres.

Ante esta dificultad, el apoyo familiar, sobre todo por parte del marido o la pareja, tanto emocional como en la colaboración con las tareas domésticas y de cuidados y de la familia política se constituye como un factor de éxito de relevancia (Reynolds et al., 2018; Saleem \& Ajmal, 2018). Las profesoras entrevistadas por Thun (2020) valoraban la flexibilidad de los horarios que ofrece el trabajo académico como un condicionante positivo. Por su parte, Fritsch (2015) señala dos tipos de estrategias adoptadas por las académicas para afrontar las dificultades de conciliación. La primera de ellas consistiría en separar la vida personal y profesional, es decir, desarrollar el trabajo de lunes a viernes, mientras que dedicarían de modo exclusivo el fin de semana a la atención y disfrute con la familia. La segunda de las estrategias trata de conciliar ambas esferas, por ejemplo, en caso de viaje o traslado a otra ciudad o país, se movilizaría toda la familia. De este modo, la posibilidad de conciliar el trabajo y la vida familiar es un indicador de éxito, condicionando el progreso profesional de las académicas (Collings et al., 2011).

Por otro lado, variables anteriores al inicio de la carrera académica limitan las posibilidades de éxito en el futuro. Jungbauer-Gans y Gross (2013) confirman, en una investigación realizada en el sistema universitario alemán, que las personas de una clase social más alta tienen mayores posibilidades de promoción. De este modo, como encontraron Gallego-Morón y Matus-López (2020) en la academia argentina, el nivel socieconómico y cultural de la familia de origen condiciona las futuras posibilidades de éxito de los individuos. Por otro lado, el tipo de modelo educativo familiar, principalmente en lo que respecta al rol paterno, en países con una marcada cultura patriarcal, influye en las trayectorias de las académicas. En el caso de Pakistán, Saleem y Ajmal (2018) encuentran que aquellas mujeres que reciben una educación paterna basada en la promoción de la autonomía, junto con el apoyo emocional, una vez iniciada la trayectoria profesional, además de aumentar los niveles de autoconfianza, repercute de un modo positivo en el éxito de sus carreras.

\section{Condicionantes grupales}

organizacionales

Existe evidencia de que la pertenencia a grupos de investigación y redes de trabajo fomenta la participación activa en la comunidad científica (Harford, 2018). El apoyo del director/a de tesis y de compañeros/as de profesión guarda una relación positiva con mayores niveles de productividad (Redmond et al., 2017; Reynolds et al., 2018). Así, la participación en programas de mentoría favorece la obtención de fondos económicos y financiación, a través de proyectos competitivos, y la realización de publicaciones de impacto (Jungbauer-Gans \& Gross, 2013; Leberman et al., 2016). El papel del mentor es esencial en tanto en cuanto desarrolla las funciones de guía y orientador, aportándoles mayor confianza y una red de trabajo y contactos (Franco-Orozco \& Franco-Orozco, 2018; Picardi, 2017; Reynolds et al., 2018).

No obstante, la academia se sigue constituyendo en la actualidad como una organización donde prevalece una cultura masculinizada (Wesarat \& Mathew, 2017). Los roles y estereotipos de género existentes en la sociedad se reproducen en el seno de la academia, en las relaciones entre profesoras y profesores. Estudios como los de Corroy y 
Savignac (2016) y Montes y O'Connor (2019) ponen de manifiesto que las académicas ven dificultadas sus trayectorias debido al trato desigual por parte de compañeros/as, la ausencia o insuficiencia de redes de apoyo y la existencia de redes informales de poder. Fritsch (2015) constata que las académicas pertenecientes a áreas tradicionalmente masculinizadas como las STEM afirman encontrar más problemas para acceder a los denominados old boy's club, esto es, tradicionales grupos de poder masculinos, lo que repercute negativamente en las posibilidades de colaboración y trabajo en equipo.

\section{Condicionantes profesionales}

\section{organizacionales}

El nivel de productividad es un factor principal en el acceso y promoción a la carrera universitaria (Jungbauer-Gans \& Gross, 2013; Thun, 2020). De este modo, la academia se constituye como un sistema meritocrático, con base en la evaluación continua del trabajo, los resultados y el reconocimiento del profesorado investigador (Montes \& O'Connor, 2019; Vayreda et al., 2019). Criterios tales como la publicación en revistas de alto impacto, la realización de estancias en el extranjero y la existencia de financiación externa repercuten de un modo positivo en el currículo investigador y las posibilidades de promoción (Reynolds et al., 2018).

A pesar de ello, la evidencia muestra que las profesoras se constituyen en menor medida como investigadoras principales de grupos de investigación y participan con menos frecuencia en la solicitud de proyectos en convocatorias competitivas (Gallego-Morón \& Matus-López, 2020). Entre aquellas que lo hacen, además, suelen recibir menor financiación (Leberman et al., 2016). Investigaciones anteriores muestran la existencia de un sesgo de género en la evaluación (Fritsch, 2015; Marini \& Meschitti, 2018; Van der Lee \& Ellemers, 2015).

De este modo, la existencia de un sistema de habilitación y promoción justo, basado en la meritocracia y objetividad se constituye como requisito previo indispensable para avanzar en la lucha contra las discriminaciones. Además, conocer el funcionamiento de ambos procesos y sus reglas del juego (Harford, 2018, p. 14), junto con procesos transparentes y abiertos, se sitúan como factores de éxito en la carrera académica (Reynolds et al., 2018).

\section{Condicionantes sociales}

Todos los factores desarrollados con anterioridad se presentan en un contexto social caracterizado por una serie de variables que en el caso de las mujeres dificultan sus posibilidades de promoción. El sorpasso femenino acontecido en las últimas décadas, en el mercado laboral, en general, y en el contexto universitario, en particular, no se ha visto acompañado del fin del modelo tradicional de la división sexual del trabajo.

Existe una amplia bibliografía que describe las características masculinizadas de las sociedades y la persistencia de estereotipos y roles de género (Cooper, 2019). La universidad no se encuentra ajena a ello, y en esta institución se aprecian rasgos similares (Gallego-Morón \& Matus-López, 2018). Aspectos como la capacidad de liderazgo o el éxito son considerados deseables y de carácter masculino (Van der Lee \& Ellemers, 2015). Por su parte, a las mujeres se les asocian capacidades menos valoradas profesionalmente, como el cuidado, la atención y la propia reproducción (Bozzon et al., 2017; Toffoletti \& Starr, 2016). Así, a la hora de promocionar, estos factores/ barreras, no afectan de igual modo a hombres y mujeres (Fritsch, 2015).

\section{Metodología}

\section{Participantes}

El estudio se realizó en la Universidad Federico II de Nápoles (Italia). El universo lo conforma el personal docente e investigador (PDI) compuesto por un total 2509 personas. El 62 $\%$ son hombres y el $38 \%$ mujeres, y por 
categoría académica, el $23 \%$ es profesorado ordinario (professore ordinario o di I fascia), el $34 \%$ profesorado asociado (professore associato o di II fascia) y el $43 \%$ profesorado investigador, total indefinido y temporal (ricercatore tipo a y tipo b). La muestra probabilística simple, estuvo compuesta por 228 participantes $(55.3 \%$ hombres y $43.7 \%$ mujeres), con un margen de error, al $95 \%$ de confianza, de 0.63. La tasa de respuesta fue del $9.1 \%$, con representación de todos los estratos por sexo, categoría académica y área de conocimiento. El rango de edad de los sujetos participantes fue de los 21 a los 67 años $(M=51.7, D E=9.2)$. En el caso de los varones, la edad promedio fue de 52.8 años $(D E=9.3)$ y de $50.3(D E=8.9)$ para las mujeres. Otros datos sociodemográficos de la muestra se observan en la Tabla 1.

Tabla 1

Datos sociodemográficos de la muestra $(N=228)$

\begin{tabular}{lrrrrrr}
\hline \multirow{2}{*}{\multicolumn{1}{c}{ Variable }} & \multicolumn{1}{c}{ Hombres } & \multicolumn{2}{c}{ Mujeres } & \multicolumn{2}{c}{ Total } \\
\cline { 2 - 8 } & $n$ & $\%$ & $n$ & $\%$ & $n$ & $\%$ \\
\hline Tramo de edad & 0 & 0 & 1 & 1 & 1 & 0.4 \\
30 & 11 & 8.7 & 11 & 10.8 & 22 & 9.6 \\
$30-39$ & 35 & 27.8 & 32 & 31.4 & 67 & 29.4 \\
$40-49$ & 40 & 31.7 & 42 & 41.2 & 82 & 36 \\
$50-59$ & 40 & 31.7 & 16 & 15.7 & 56 & 24.6 \\
$>60$ & & & & & & \\
Estado Civil & 13 & 10.3 & 23 & 22.5 & 36 & 15.8 \\
Soltero/a & 100 & 79.4 & 62 & 60.8 & 162 & 71.1 \\
En pareja & 13 & 10.3 & 17 & 16.7 & 30 & 13.2 \\
Separado/a y Viudo/a & & & & & & \\
Categorín académica & 44 & 34.9 & 19 & 18.6 & 63 & 27.6 \\
Profesorado ordinario & 42 & 33.3 & 40 & 39.2 & 82 & 36 \\
Profesorado asociado & 40 & 31.7 & 43 & 42.2 & 83 & 36.4 \\
Profesorado Investigador & & & & & & \\
Area de conocimiento & 36 & 28.6 & 36 & 35.3 & 72 & 31.6 \\
Humanidades y Sociales & 8 & 6.3 & 12 & 11.8 & 20 & 8.8 \\
Agraria y Veterinaria & 25 & 19.8 & 17 & 16.7 & 42 & 18.4 \\
Medicina y Cirugía & 57 & 45.2 & 37 & 36.3 & 94 & 41.2 \\
Politécnica y Cs. Básicas & 126 & 55.3 & 102 & 44.7 & 228 & 100 \\
\hline Total & & & & & & \\
\hline & & & & & &
\end{tabular}

\section{Instrumento}

Para medir la percepción de los factores de éxito y las barreras en la carrera académica, se utilizó un método de corte cuantitativo con base en una encuesta ad hoc validada por personas expertas en la materia pertenecientes a la Universidad Pablo de Olavide (España) y la Universidad Federico II (Italia), diseñada con base en la revisión de la bibliografía científica existente (GallegoMorón \& Matus-López, 2018; Gallego-Morón et al., 2020; Guil, 2004) y utilizada previamente en el contexto universitario argentino (GallegoMorón, 2018).

Este instrumento está compuesto por 30 ítems, estructurados en dos escalas. La primera de ellas sobre factores de éxito compuesta por 16 ítems (tres personales cognitivo-emocionales; cinco personales familiares; cuatro organizacionales grupales; cuatro organizaciones profesionales) y la segunda por 14 ítems sobre barreras a la promoción (tres personales cognitivoemocionales; tres personales familiares; tres organizacionales grupales; dos organizaciones profesionales; tres sociales). Estas fueron expresadas de forma afirmativa y se solicitó a las personas encuestadas que valorasen en una escala Likert de 1 a 5 la importancia que los condicionantes habían tenido o tenían en la actualidad en su propia carrera académica. Una puntuación más alta indica una mayor importancia percibida (Apéndice A).

\section{Procedimiento}

El cuestionario se realizó por vía electrónica, con respuesta anonimizada. La captación fue a través de enlaces enviados por el equipo investigador a los correos electrónicos oficiales del total del PDI de la Federico II, donde se describía el objetivo del estudio, se solicitaba el consentimiento para el uso de los datos recogidos y se garantizaba la confidencialidad y anonimato. El estudio tuvo lugar durante el mes de octubre de 2018. Las respuestas de la encuesta fueron vaciadas en una sábana de datos del programa de análisis estadístico IBM SPSS (v.25). Se utilizaron las variables de sexo (hombre, mujer), categoría académica $^{1}$ (ordinario, associato y ricercatore), factores de éxito (e01 a e14) y barreras a la promoción (b01 a b16). La explotación de la 
encuesta se realizó a través de tres instrumentos. En primer lugar, un análisis estadístico de las valoraciones y significancia de medias por sexo (prueba $t$ ). En segundo lugar, un análisis de correlaciones entre factores de éxito y barreras y, en tercer lugar, un análisis de correspondencias múltiples.

\section{Resultados}

\section{Análisis descriptivo de valoraciones medias}

La valoración media de los factores de éxito es más alta que la de las barreras a la promoción, pero con diferencias por sexo (Apéndice B). Mujeres y hombres valoran más los factores (3 sobre 5 en ambos sexos), pero las primeras puntúan las barreras un $30 \%$ más que los hombres ( 2.6 y 2 , respectivamente).

Los factores de éxito más importantes para ambos sexos son la vocación y motivación (e01), capacidad intelectual (e02) y constancia y trabajo (e03) (Figura 1). En las barreras coinciden las dos más valoradas: la existencia de redes informales de poder (b08) y la dificultad para conciliar vida personal, familiar y laboral (b01). No obstante, en otras cuatro barreras las mujeres asignan entre $1 \mathrm{y}$ 1.5 puntos más de importancia que los hombres: sesgo de género en la evaluación (b09), cultura machista en la universidad (b12), estereotipos y roles de género (b13) y sociedad patriarcal (b14).
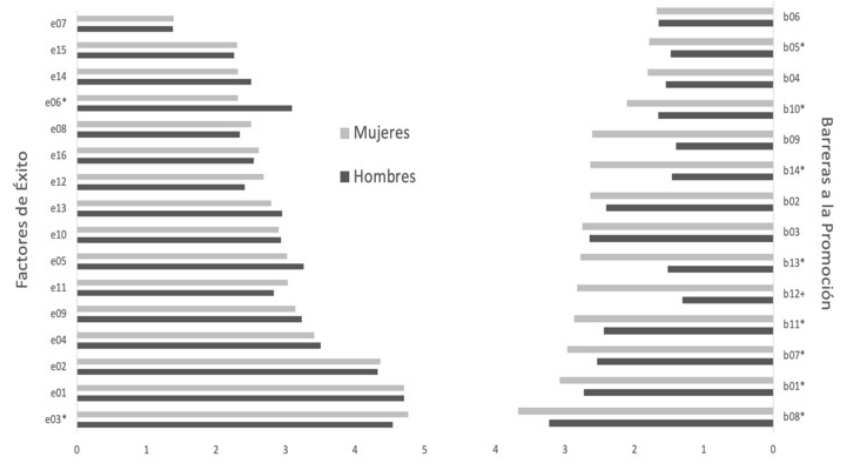

Figura 1

Valoraciones medias de factores de éxito (e) y barreras (b) según sexo

$$
\begin{aligned}
& \text { *Diferencias por sexo } \\
& \text { significativas al 95\% de confianza. }
\end{aligned}
$$

Análisis de correlaciones entre factores de éxito y barreras

Las correlaciones par-a-par de los factores de éxito y las barreras entregan estadísticos significativos en 60 de los 224 cruces (Apéndice C). Todos los factores tienen correlación significativa con alguna barrera, y solo una barrera no muestra correlación significativa con ningún factor de éxito (sociedad patriarcal, b14). Estos resultados permiten constituir cinco grupos de relaciones, que serán la base de los modelos analizados. Cada grupo de relaciones tiene la siguiente constitución:

- Grupo 1. Constituido por variables que mantienen relación principalmente con el ámbito personal cognitivo-emocional. Resulta de la relación entre los factores de éxito como son la vocación y motivación (e01), la capacidad intelectual (e02) y la constancia y el trabajo (e03), con las barreras como el bajo nivel de autoestima (b04), la falta de confianza en sí mismo/a (b05) y decisión personal de no promocionar (b06). Sus correlaciones son negativas en todos los casos, con un rango de -0.266 hasta -0.04 , con significancia en el $77 \%$ de los cruces.

- Grupo 2. Constituido por variables que dicen relación principalmente con el ámbito personal familiar en los factores de éxito y organizacionales 
en las barreras. Resulta de la relación entre los factores de apoyo familiar (e04), apoyo de la pareja (e05) y facilidad de conciliación (e06), con las barreras de trato desigual de los pares (b07), redes informales de poder (b08), sesgo de género en la evaluación de méritos (b09) y falta de una red de apoyo (b10). Sus correlaciones son negativas en todos los casos, con un rango de -0.258 hasta -0.035 , con significancia en el $83 \%$ de los cruces.

- Grupo 3. Constituido por variables que guardan relación principalmente con el ámbito organizacional. Resulta de la relación entre los factores de éxito mentoring (e11), realización de publicaciones colectivas (e12), estancias en el extranjero (e13), financiación externa (e14), sistema de acreditación objetivo (e15) y sistema de concurso objetivo (e16), y las barreras de trato desigual de los pares (b07), redes informales de poder (b08) y falta de una red de apoyo (b10). Sus correlaciones son negativas en todos los casos, con un rango de -0.292 hasta -0.086 , con significancia en el $83 \%$ de los cruces.

- Grupo 4. Constituido por variables que dicen relación principalmente con el ámbito organizacional grupal en los factores de éxito y personal familiar en las barreras. Resulta de la relación entre los factores como "estar en el momento justo en el lugar adecuado" (e09), pertenencia a grupo de investigación (e10), mentoring (e11) y realización de publicaciones colectivas (e12), y las barreras de trabajo doméstico y de cuidados (b02) y falta de tiempo (b03). Sus correlaciones son positivas en todos los casos, con un rango de 0.141 hasta 0.211 , con significancia en el $100 \%$ de los cruces.

- Grupo 5. Constituido por variables relevantes que reflejan desigualdades explícitas, pero que están escasamente relacionadas con otros condicionantes. Estos son factores de éxito como la presencia de académicos en la familia (e07) y el nivel socioeconómico de la familia de origen (e08), y las barreras como la dificultad para conciliar vida personal, familiar y laboral (b01), cultura machista en la universidad (b12), estereotipos y roles de género (b13) y sociedad patriarcal (b14). Sus correlaciones son negativas en la mitad de los casos, con un rango de -0.157 hasta 0.134 , con significancia en el $25 \%$ de los cruces.

\section{Análisis de correspondencias múltiples}

Los grupos anteriores fueron la base para la construcción de los modelos que se sometieron al análisis de correspondencias múltiples. Para ello, las categorías de las variables de barreras y factores de éxito fueron reclasificadas, de menor a mayor importancia, en valores de: Baja (1), Media (2) y Alta (3) ${ }^{2}$. Se mantuvo la clasificación por sexo y categoría académica. Esto permite que las dimensiones del modelo estén construidas principalmente sobre barreras y factores, siendo más efectivo el análisis visual de la ubicación de las categorías de ambas variables.

El análisis gráfico de cada modelo se presenta a continuación. El resumen, para cada modelo, de los estadísticos de fiabilidad (alfa de Cronbach e Inercia), así como de las dimensiones (medidas discriminantes) están recogidos en el Apéndice D.

\section{Modelo 1. Personal Cognitivo-Emocional}

Los cuadrantes quedan relativamente organizados según la valoración de factores de éxito y de barreras (Figura 2$)^{3}$. La dimensión 1 (27.5 \% de varianza y $62.4 \%$ de $\alpha$ de Cronbach), está relativamente más determinada por las barreras: bajo nivel de autoestima (b04) y falta de confianza en sí mismo/a (b05). Por su parte, la segunda dimensión (26.3 \% de varianza y $60 \%$ de $\alpha$ de Cronbach) queda determinada por los factores de éxito: capacidad intelectual (e02) y trabajo, constancia y perseverancia (e03).

En esta ordenación se pueden observar dos cuestiones. Primero, la categoría de profesorado ordinario parece quedar diferenciada de las otras categorías académicas de profesorado asociado (prof. associato) e investigador (ricercatore), situándose en el cuadrante con valoraciones altas de los factores de éxito y valoraciones bajas de las barreras individuales. En segundo lugar, parece existir poca distancia entre las categorías 
de sexo. Sin embargo, quedan posicionadas en distintos cuadrantes, lo que deja a las mujeres relativamente más cerca de las puntuaciones altas de las barreras bajo nivel de autoestima (b04) y falta de confianza en sí mismo/a (b05).
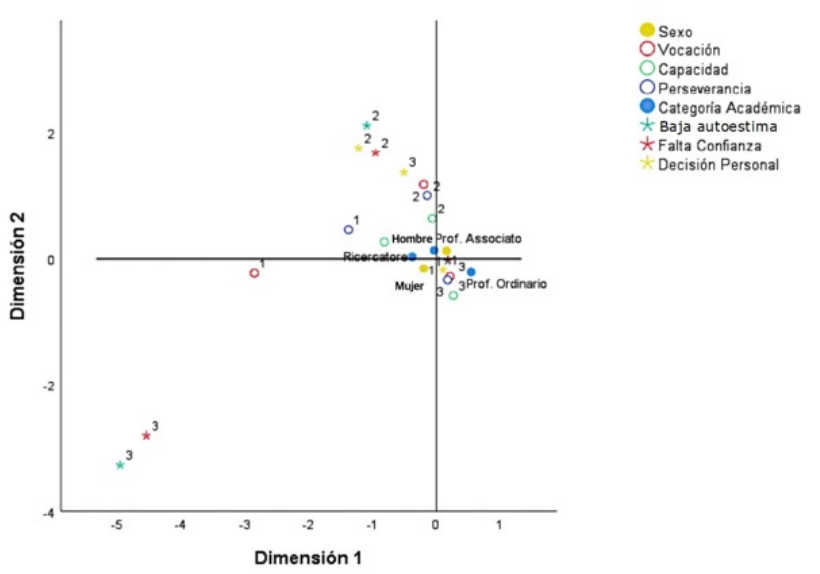

Figura 2

Resultado gráfico del análisis de correspondencias múltiples del Modelo 1

\section{Modelo 2. Personal Familiar/Organizacional}

$\mathrm{Al}$ igual que el modelo anterior, las dimensiones permiten posicionar las categorías en función de la valoración de barreras (dimensión 1) y de factores de éxito (dimensión 2). La dimensión 1 (29.8 \% de varianza y $70.6 \%$ de $\alpha$ de Cronbach) está más determinada por las barreras organizacionales grupales: trato desigual de pares (b07), redes informales de poder (b08) y falta de red de apoyo (b10). Por otro lado, la segunda dimensión (20.5\% de varianza y $51.5 \%$ de $\alpha$ de Cronbach), está determinada por los factores éxito personales familiares: apoyo familiar (e04) y apoyo de la pareja (e05).

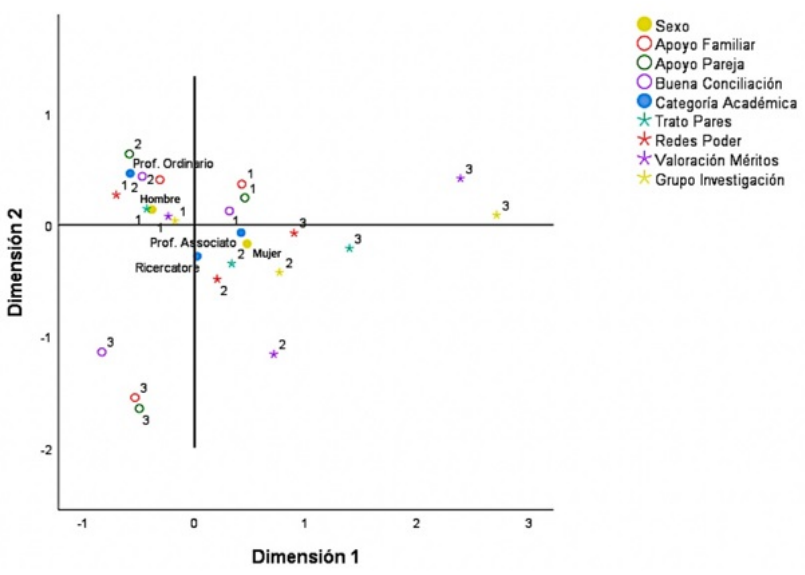

Figura 3

Resultado gráfico del análisis de correspondencias múltiples del Modelo 2

De este análisis, se obtienen cuatro conclusiones. En primer lugar, se aprecia una mayor distancia entre mujeres y hombres, situándose en cuadrantes diferentes. De este modo, las primeras están más cerca de las valoraciones medias y altas de las barreras (Figura 3). En segundo lugar, la categoría de profesorado ordinario se distancia de las otras categorías y se sitúa más cerca de los hombres que de las mujeres, con bajas valoraciones de las barreras. Como contrapartida, el resto del profesorado se encuentra más cerca de las mujeres y de las valoraciones altas de las barreras.

En tercer lugar, hombres y la categoría de profesorado ordinario se sitúan ligeramente más cerca de la valoración media/alta de los factores de éxito. Por su parte, las mujeres y las otras categorías académicas se posicionan relativamente más cerca de la valoración baja de factores de éxito. Por último, las valoraciones medias y altas de las redes informales de poder se ubican más cerca de mujeres y de las categorías académicas de profesorado asociado (prof. associato) e investigador (ricercatore). Una evidencia relevante siendo esta la barrera con mayor valoración media. 


\section{Modelo 3. Organizacional}

La dimensión 1 del modelo (24.9 \% de varianza y $69.9 \%$ de $\alpha$ de Cronbach) está más determinada por los factores de éxito: estancias en el extranjero (e13) y financiación externa (e14). En tanto, la dimensión $2(17.7 \%$ de varianza y $53.5 \%$ de $\alpha$ de Cronbach), está determinada relativamente más por el factor de éxito de mentoring (e11) y la barrera de falta de financiación (b11), coherentemente con la dimensión 1. En el análisis conjunto, se aprecia la concentración de las valoraciones altas de las barreras en el cuadrante superior derecho y la valoración alta de los factores de éxito en el superior izquierdo (Figura 4). En términos de categoría académica, el profesorado ordinario se aleja del resto, acercándose relativamente más a las valoraciones medias y altas de los factores de éxito y a las bajas de las barreras. En el cuadrante opuesto se localiza la categoría de profesorado asociado (prof. associato) e investigador (ricercatore), relativamente más cerca de las valoraciones altas de las barreras y bajas de los factores.

En relación al sexo, hombres y mujeres se ubican de forma similar a las categorías. Es decir, en cuadrantes diferentes, pero con menor distancia entre estas. Así, las conclusiones son similares, con una ubicación de hombres más parecida a profesorado ordinario y mujeres más cerca de la categoría personal investigador.

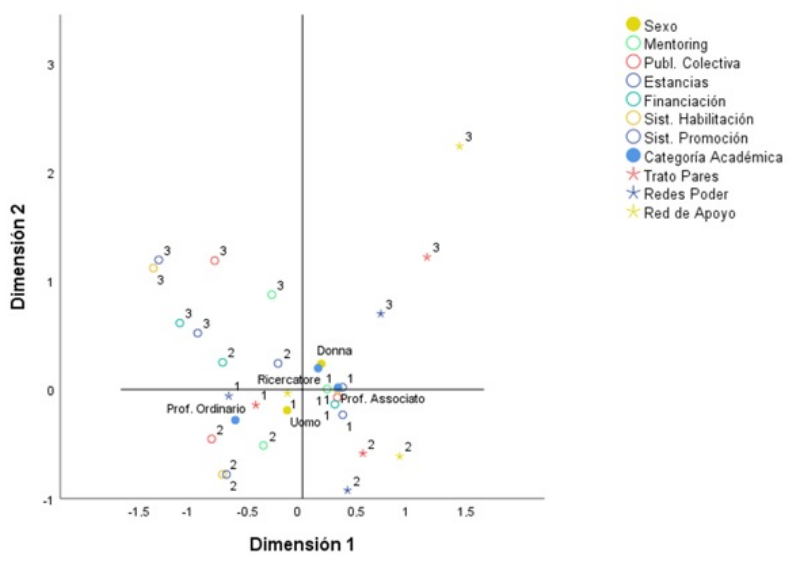

Figura 4

Resultado gráfico del análisis de correspondencias múltiples del Modelo 3

\section{Modelo 4. Organizacional Grupal/Personal Familiar}

La dimensión 1 (28.9 \% de varianza y $64.9 \%$ de $\alpha$ de Cronbach) está más determinada por el factor de éxito de pertenencia a grupos de investigación (e10) y la capacidad para "estar en el momento justo en el lugar adecuado" (e09). Por su parte, la segunda dimensión (21.2\% de varianza y $47 \%$ de $\alpha$ de Cronbach) quedó determinada principalmente por el factor de éxito mentoring (e11).

El análisis conjunto presenta agrupaciones similares de barreras y factores de éxito según la valoración. Los valores bajos de las primeras se sitúan cerca de los valores bajos de los segundos, los valores medios cerca de los medios y los altos con los altos (de unas y otros). Algo que no se produce tan claramente en los otros modelos (Figura 5). De esta manera, las categorías de profesorado asociado (prof. associato) y hombres se ubican más cerca de las valoraciones bajas de barreras y factores, mientras que mujeres y el personal investigador (ricercatore) se sitúan más cerca de las valoraciones altas. Por último, la categoría profesorado ordinario se aleja de las otras categorías académicas y se aproxima relativamente más a las valoraciones medias (valor 2). 


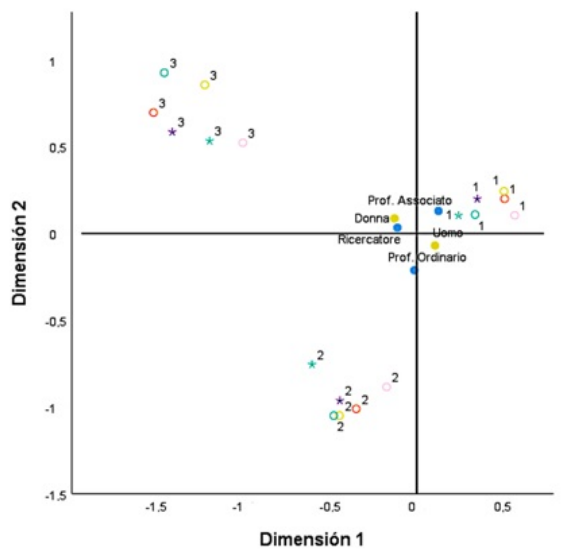

Figura 5

Resultado gráfico del análisis de correspondencias múltiples del Modelo 4

\section{Modelo 5. Desigualdades}

La primera dimensión (34.5 \% de varianza y $72.9 \%$ de $\alpha$ de Cronbach) está más determinada por la barrera de dificultad para conciliar vida personal, familiar y laboral (b01). Por su parte, en la segunda dimensión ( $25.3 \%$ de varianza y 57.9 $\%$ de $\alpha$ de Cronbach), el principal determinante es el factor de éxito de presencia de académicos en la familia (e07).
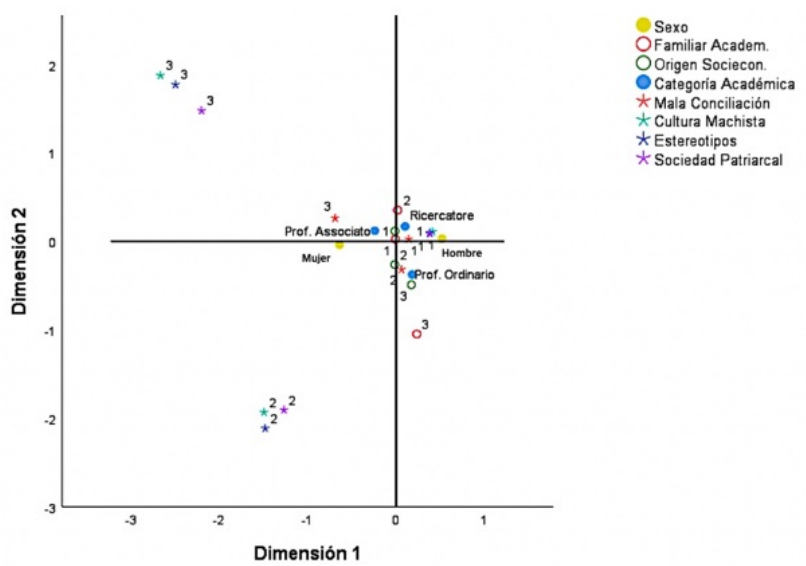

Figura 6

Resultado gráfico del análisis de correspondencias múltiples del Modelo 5

En el análisis conjunto, se refleja la relación cercana de las valoraciones medias y altas de las barreras que tiene relación directa con el género como son la cultura machista en la universidad (b12), estereotipos y roles de género (b13) y sociedad patriarcal (b14) (Figura 6). Estas características se relacionan con la mayor distancia que separa las categorías de sexo. Así, las mujeres se ubican más cerca de estas valoraciones, y por el contrario, los hombres se sitúan relativamente más cerca de las valoraciones bajas de las barreras. Por último, aunque menos evidente, la categoría académica profesorado ordinario se encuentra más cerca de las valoraciones altas de los factores de éxito, en comparación con las otras categorías académicas.

\section{Discusión}

Esta investigación, a diferencia de otras realizadas previamente a nivel internacional y, de manera particular, en Italia, no solo confirma que diferentes condicionantes son percibidos en mayor o menor medida como factores de riesgo o de éxito por el profesorado; también identifica la tipología de estos, la intensidad con la que son percibidos y que dicha relación está moderada por variables, como el sexo y la categoría académica. Los resultados muestran la carrera académica como un laberinto de cristal, esto es, como un entramado de factores de diversa índole que facilitan o dificultan las trayectorias del personal docente e investigador.

En consonancia con estudios anteriores (Franco-Orozco \& Franco-Orozco, 2018; Gallego-Morón \& Matus-López, 2020; Redmond et al., 2017), en el camino hacia el éxito se confirma la importancia de condicionantes personales como la motivación, el esfuerzo y la capacidad intelectual, valorados altamente por todo el profesorado en su conjunto. Frente a ello, factores provenientes de la propia universidad, como la existencia de redes informales de poder (Fritsch, 2015; Montes \& O'Connor, 2019), seguido por las dificultades derivadas de la conciliación personal, familiar y laboral (Bozzon et al., 2017; Bonache et al., 2020), son percibidos como los principales obstáculos que se deben solventar. 
Sin embargo, en línea con los hallazgos de otros estudios mencionados en la introducción (Collings et al., 2011; European Commission, 2019; Reynolds et al., 2018), existen diferencias por género. Por una parte, todas las barreras tienen un mayor impacto en las mujeres que en los hombres, siendo valoradas un $30 \%$ más $y$ encontrando en nueve de ellas diferencias significativas. Por otra parte, las barreras que explícitamente identifican desigualdades de género, como una cultura machista, la existencia de estereotipos de género y una sociedad patriarcal, son casi omitidas por los hombres, quienes otorgan entre 1 y 1.5 puntos menos. Esta cuestión es comprensible, ya que los varones no padecen las consecuencias directas del latente modelo dicotómico que diferencia entre ámbito masculino/productivo y femenino/reproductivo. En una sociedad como la italiana, con una fuerte tradición familista, una de cada cuatro personas sigue considerando que el rol de la mujer en la sociedad es el de ser buenas amas de casa y esposas (Emes, 2017). Además, se encontró que estas barreras no se correlacionan con ningún factor de éxito. Ello supone una cuestión de interés, ya que la desventaja social que las académicas encuentran no consigue ser compensada con ningún otro factor.

El principal aporte de este estudio reside en la identificación de los condicionantes que son percibidos de manera diferenciada como factores de éxito u obstáculos, de acuerdo al género y al rango ocupado. Los resultados señalan que las mujeres suelen mostrar comportamientos en la misma línea que el profesorado asociado y el personal investigador, mientras que los hombres se comportan más como el profesorado ordinario. No se trata solo de que las mujeres están menos representadas en la categoría más alta, sino que, incluso excluyendo esta variable, las diferencias son relevantes. Así, las mujeres y las categorías media y baja otorgan una mayor relevancia a las barreras organizacionales, mientras que los hombres y la categoría más alta perciben más la importancia de los factores de éxito personales y familiares.

En esta línea, se identifican tres resultados que complementan la evidencia empírica de estudios anteriores, en relación con el papel que desempeñan las redes informales de poder, la conciliación y los condicionantes grupales. En primer lugar, el análisis de correspondencias múltiples de las barreras organizacionales permite visualizar que las redes informales de poder no afectan por igual a todo el profesorado. De esta manera, la valoración alta de esta barrera se relaciona más con las mujeres y con las categorías media y baja. Estos resultados contrastan con estudios previos en los que el profesorado insiste en la objetividad y meritocracia en el ámbito universitario (Gallego-Morón \& MatusLópez, 2018). No hay que olvidar, no obstante, que las académicas italianas se concentran en mayor medida que los varones entre el personal investigador ( $23 \%$ para las mujeres y 17.6 $\%$ para los hombres) (MIUR, 2020). Además, desde una aproximación feminista, podemos confirmar que el poder no es ajeno al género en una sociedad patriarcal donde, a nivel global, los varones dominan las cotas de poder en todos los ámbitos productivos (judicial, ejecutivo, legislativo, militar, económico, religioso, cultural y científico), así como en el reproductivo.

Desde la Psicología Social y de las Organizaciones el interés por analizar el tema del poder en el ámbito laboral, en general, y más recientemente, en la universidad, en particular, es amplio (Guil, 2004). La existencia de coaliciones de hombres como táctica para conseguir permanecer en su posición privilegiada, hace que las redes informales de poder afecten de especial manera al profesorado que ocupa posiciones inferiores, pero sobre todo a las mujeres, quienes se constituyen como sujetos que tradicionalmente no han formado parte del "club". Esta situación, combinada con la existencia de procedimientos formales de reclutamiento nominativo, esto es, sin necesidad de convocatoria de concurso público, como es el caso de la "llamada directa" (chiamata diretta), hacen cuestionar la supuesta meritocracia del sistema universitario. En esta línea, estudios como los de Marini y Meschitti (2018), en el contexto italiano, muestran que, en igualdad de producción científica, los hombres tienen aproximadamente un $24 \%$ más de probabilidades 
de ser promocionados. Por su parte, tras analizar 2823 candidaturas a una prestigiosa beca en los Países Bajos, Van der Lee y Ellemers (2015) concluyeron la existencia de un sesgo de género a favor de los solicitantes varones. En proyectos igualmente valorados, existía una preferencia hacia la elección del candidato masculino.

En segundo lugar, los resultados también aportan una nueva evidencia sobre las consecuencias de la conciliación de la vida personal, familiar y laboral. La literatura ha señalado ampliamente que los cuidados y las tareas domésticas siguen siendo considerados en el imaginario colectivo como una cuestión femenina (Bonache et al., 2020; Gorshkova \& Miryasova, 2020; Toffoletti \& Starr, 2016). Por su parte, los varones se encuentran liberados de las tareas reproductivas. Ante esta escasa o inexistente corresponsabilidad masculina, las mujeres deben asumir una segunda jornada laboral, con consecuencias directas en la disponibilidad de tiempo, dedicación y movilidad, y por ende, en las posibilidades de promoción (Castañeda-Rentería et al., 2019). Los estudios sobre la temática habitualmente se centran en el análisis de tal condicionante, teniendo como objeto de estudio a las mujeres (Collings et al., 2011; Fritsch, 2015; Reynolds et al., 2018; Thun, 2020). Sin embargo, en la presente investigación se analiza la influencia negativa o positiva de este ítem tanto para hombres como mujeres. De este modo, se constata que "la facilidad para conciliar la vida personal, familiar y laboral" se comporta como un factor de éxito para los varones y el profesorado ordinario.

Los hombres parecen contar con más apoyos familiares para dar respuesta a esa necesidad, consistentes, en la mayor parte de las ocasiones, en la asunción u organización de las responsabilidades domésticas o de cuidados por parte de sus esposas/parejas. Así, sí existe una contrapartida de esta variable, el aumento de la corresponsabilidad (que puede ser positiva e impulsar la carrera laboral), frente a su escasez o ausencia, que en la actualidad está frenando las posibilidades de éxito de las mujeres. En conclusión, lo que para unas se presentan como uno de los mayores obstáculos por solventar en el camino al éxito, para otros, además, se constituye como un condicionante positivo.

En tercer lugar, son de especial interés los resultados mostrados en el Modelo 4 de análisis de correspondencias múltiples. Se observa una relación entre los factores de éxito organizacionales grupales y la existencia de dificultades personales familiares. Así, las mujeres y el personal investigador se sitúan más cerca de las valoraciones altas de ambas tipologías de condicionantes. Por su parte, los varones y el profesorado asociado otorgan una menor relevancia a estos. De esta manera, frente a los problemas de conciliación y la falta de tiempo derivada de ello, las académicas y el profesorado base encuentran una respuesta en el trabajo grupal. La pertenencia a grupos de investigación, el mentoring y la realización de publicaciones colectivas se constatan como las principales estrategias para suplir la menor disponibilidad laboral.

\section{Conclusiones}

El presente estudio permite avanzar en el conocimiento de los factores que condicionan la participación del personal docente e investigador en la carrera académica en tres aspectos: (1) el análisis se centra en los obstáculos y los factores de éxito y sus posibles interconexiones; (2) enfatiza el aspecto innovador de las diferencias en las percepciones por sexo y categorías académicas, que hasta el momento ha recibido poca atención. Mientras que, en otros estudios, se analiza de modo aislado las percepciones de las mujeres o de profesorado perteneciente a ciertas categorías, en este trabajo, se realiza un análisis de correspondencia múltiple que abarca todas las variables; (3) se avanza en la relevancia de los factores de éxito y obstáculos en cada una de las etapas universitarias, constituyendo un diagnóstico previo necesario para el diseño y aplicación de medidas de intervención dirigidos a diferentes colectivos.

Con el principal reto de contribuir a cerrar esta brecha en la investigación existente, este 
trabajo explora los condicionantes personales, organizacionales y sociales más estudiados en la literatura, lo que permite comparar la situación de la Universidad Federico II con otras universidades italianas y del mundo. Sus implicaciones pueden servir para el diseño de medidas que apoyen la igualdad de oportunidades en la carrera científica.

El compromiso de la comunidad internacional por lograr la igualdad de género es un objetivo prioritario, como recoge la Agenda 2030 para el Desarrollo Sostenible de las Naciones Unidas. Desde hace años, en el ámbito científico e investigador se están tomando medidas para favorecer la igualdad de género en la promoción del profesorado, para, en definitiva, promover el mérito con justicia. Sin embargo, existen diferencias notables en el compromiso adoptado por los gobiernos y las instituciones universitarias de los diferentes países. Como se deriva de este trabajo, la cuestión debe ser abordada teniendo en consideración la multidimensionalidad y estructuralidad. Así, existen factores que exceden el ámbito académico y otros que determinan las estructuras organizacionales. Pero también existe un margen de intervención para visualizar la problemática, fomentar los factores de éxito y reducir las barreras discriminatorias en el camino de mujeres y hombres de todos los rangos hacia la cima académica.

Algunas limitaciones inherentes a la metodología implementada deben ser tenidas en cuenta a la hora de extrapolar los resultados del presente estudio. Primero, el tamaño de la muestra estudiada, a pesar de no ser pequeño, no es representativo de la población de todo el profesorado universitario existente en Italia. No obstante, la muestra se encuentra dentro de los parámetros de anteriores estudios de casos. Segundo, aunque el cuestionario fue enviado al total del universo, las personas participantes podrían haberse autoseleccionado de manera sesgada. No es descartable que la invitación haya sido aceptada mayoritariamente por aquellas personas que tienen una mayor sensibilidad hacia temas de género e igualdad. Sin embargo, los resultados presentados forman parte de una investigación más amplia donde se combinan técnicas cuantitativas y cualitativas, produciéndose la validación de los resultados a través de la triangulación metodológica.

Por último, sería importante llevar a cabo investigaciones longitudinales que profundicen en el análisis de las percepciones de los condicionantes a mediano y largo plazo. También, convendría profundizar en las relaciones existentes entre el género, la categoría académica y la disciplina o campo de conocimiento. Otro aspecto interesante sería el análisis del impacto de las medidas que se están llevando a cabo, como los planes de igualdad, la implementación de cuotas en los tribunales de evaluación o las reformas de los sistemas de acreditación del profesorado.

\section{Referencias}

Bonache, H., Carballo, V., Chas, A., \& Delgado, N. (octubre, 2020). Go the extra mile: A gender approach towards parenthood in early-career academics. Journal of Gender Studies. https://doi.org/10.1080/09589236. 2020.1836482

Bozzon, R., Murgia, A., Poggio, B., \& Rapetti, E. (2017). Work-life interferences in the early academic career stages: The case of precarious researchers in Italy. European Educational Research Journal, 16(2-3), 332-351. https://doi.org/10.1177/1 474904116669364

Castañeda-Rentería, L. I., Contreras, K. A., \& Parga, M. F. (Coords.). (2019). Mujeres en las universidades iberoamericanas: la búsqueda de la necesaria conciliación trabajo-familia. Universidad de Guadalajara.

Collings, S., Conner, L., McPherson, K., Midson, B., \& Wilson, C. (2011). Learning to be leaders in higher education: What helps or hinders women's advancement as leaders in universities. Educational Management Administration E⿱ Leadership, 39(1), 44-62. h ttps://doi.org/10.1177/1741143210383896

Conferenza dei Rettori delle Università italiane. (2020). Elenco Rettori. 
Autor. https://www.crui.it/atenei-e-rettoricrui/elenco-rettori.html

Cooper, O. (2019). Where and what are the barriers to progression for female students and academics in UK Higher Education? Perspectives: Policy and Practice in Higher Education, 23(2-3), 93-100. https://doi.org/ 10.1080/13603108.2018.1544176

Corroy, L., \& Savignac, E. (2016). Female academics in France and the glass ceiling organized scarcity? Alternative Politics/ Alternatif Politika, 22-40. https://hal.archive s-ouvertes.fr/hal-01423421

European Commission. (2019). She figures 2018. Publications Office of the European Union. https://ec.europa.eu/info/publicatio ns/she-figures-2018_en

Franco-Orozco, C. M., \& Franco-Orozco, B. (2018). Women in science: An overview of the challenges towards gender equality in Colombia and how to move forward. Frontiers in Astronomy and Space Sciences, 5(24), 1-12. https://doi.org/10.3389/fspas.2 018.00024

Fritsch, N. S. (2015). At the leading edge-does gender still matter? A qualitative study of prevailing obstacles and successful coping strategies in academia. Current Sociology, 63(4), 547-565. https://doi.org/10.1177/00 11392115576527

Gallego-Morón, N. (2018). Discriminación de género en el sistema universitario argentino. Revista Estudos Feministas, 26(2), 1-18. https://doi.org/10.1590/1806-9584-2 $018 \mathrm{v} 26 \mathrm{n} 251339$

Gallego-Morón, N., \& Matus-López, M. (2018). Techo de cristal en las universidades españolas. Diagnóstico y causas. Profesorado: Revista de Currículum y Formación del Profesorado, 22(3), 209-229. https://doi.org/10.30827/profesor ado.v22i3.7999

Gallego-Morón, N., \& Matus-López, M. (2020). Factores positivos en las trayectorias de las académicas e investigadoras argentinas. Cuestiones de género: de la igualdad y la diferencia, 15, 105-124. https://doi.org/10.1 8002/cg.v0i15.6174
Gallego-Morón, N., Matus-López, M., \& Gálvez, L. (2020). Revisión sistemática de la literatura sobre el fenómeno del techo de cristal en las universidades españolas. Revista Iberoamericana de Educación Superior, 11(31), 130-149, https://doi.org/1 0.22201/iisue.20072872e.2020.31.710

Gorshkova, I. D., \& Miryasova, O. (2020). Gender inequality in current Russian higher education institutions. Woman in Russian Society, 1, 29-44. https://doi.org/10.21064/ WinRS.2020.1.3

Guil, A. (2004). Techos de cristal en la Universidad de Sevilla. Ministerio de Trabajo y Asuntos Sociales.

Harford, J. (2018). The perspectives of women professors on the professoriate: A missing piece in the narrative on gender equality in the university. Education Sciences, 8(50), 1-17. https://doi.org/10.3390/educsci80200 50

Instituto de Estadística de la UNESCO. (2018). UNESCO eAtlas of Gender Inequality in Education. Autor. http://uis.unesco.org

Instituto de Estadística de la UNESCO. (2020). Women in science (Fact Sheet N. - 60). http:// uis.unesco.org/sites/default/files/document s/fs60-women-in-science-2020-en.pdf

Emes, C. (2017). The changing role of women? Ipsos Global Trends. https://www.ipsosglobaltrends.com /the-changing-role-of-women/

Jungbauer-Gans, M., \& Gross, C. (2013). Determinants of success in university careers: Findings from the German academic labor market. Zeitschrift für Soziologie, 42 (1), 74-92. https://doi.org/10.1 515/zfsoz-2013-0106

Leberman, S. I., Eames, B., \& Barnett, S. (2016). Unless you are collaborating with a big name successful professor, you are unlikely to receive funding. Gender and Education, 28(5), 644-661. https://doi.org/10.1080/09 540253.2015.1093102

Marini, G., \& Meschitti, V. (2018). The trench warfare of gender discrimination: Evidence from academic promotions to full professor 
in Italy. Scientometrics, 115(2), 989-1006. h ttps://doi.org/10.1007/s11192-018-2983-4

Ministero dell'Istruzione dell'Università e della Ricerca. (2020). Portale dei dati dell'istruzione superiore. Ufficio Gestione Patrimonio Informativo e Statistica. http://ustat.miur.i t/dati/didattica/italia/atenei

Montes, E., \& O'Connor, P. (2019). Micropolitics and meritocracy: Improbable bed fellows? Educational Management Administration $\mathcal{E}$ Leadership, 47(5), 678-693. https://doi.org/ $10.1177 / 1741143218759090$

Morrison, A. M., White, R. P., \& Van Velsor, E. (1987). Breaking the glass ceiling. AddisonWesley.

Obiol, S., \& Villar, A. (2016). Locas por las ciencias. Las dificultades de rendir cuentas y cuidar. Mètode, 92, 37-42. https://doi.org/ 10.7203/metode.7.8080

Picardi, I. (2017). La dimensione di genere nelle carriere accademiche. Federico II University Press.

Rebolledo, L., \& Espinoza, M. P. (2016). Género, universidad e investigación. Una tríada compleja. Revista Anales, 11, 157-171. http s://doi.org/10.5354/0717-8883.2017.45234

Redmond, P., Gutke, H., Galligan, L., Howard, A., \& Newman, T. (2017). Becoming a female leader in higher education: Investigations from a regional university. Gender and Education, 29(3), 332-351. https://doi.org/10.1080/09540253 .2016 .1156063

Reynolds, A. C., O’Mullan, C., Pabel, A., MartinSardesai, A., Alley, S., Richardson, S., Colley, L., Bousie, J., \& McCalman, J. (2018). Perceptions of success of women early career researchers. Studies in Graduate and Postdoctoral Education, 9(1), 2-18. http s://doi.org/10.1108/SGPE-D-17-00019

Saleem, G., \& Ajmal, M. A. (2018). Workfamily balance: Experiences of successful professional women in academia in Pakistan. Pakistan Journal of Psychological Research, 33(1), 101-121. https://www.rese archgate.net/publication/329752463_Wor $\mathrm{k}$-family_balance_Experiences_of_successf ul_professional_women_in_academia_in_ Pakistan

Thun, C. (2020). Excellent and gender equal? Academic motherhood and 'gender blindness' in Norwegian academia. Gender, Work E Organization, 27(2), 166-180. http s://doi.org/10.1111/gwao.12368

Toffoletti, K., \& Starr, K. (2016). Women academics and work-life balance: Gendered discourses of work and care. Gender, Work $\&$ Organization, 23(5), 489-504. https://doi.org/10.1111/gwao.121 33

Van der Lee, R., \& Ellemers, N. (2015). Gender contributes to personal research funding success in The Netherlands. PNAS, 112(40), 12349-12353. https://doi. org/10.1073/pnas.1510159112

Vayreda, A., Conesa, E., Revelles-Benavente, B., \& González, A. M. (2019). Subjectivation processes and gender in a neoliberal model of science in three Spanish research centres. Gender, Work \& Organization, 26(4), 430-447. https://doi.org/10.1111/gw ao. 12360

Wesarat, P. O., \& Mathew, J. (2017). Theoretical framework of glass ceiling: A case of India's women academic leaders. Paradigm, 21 (1), 21-30. https://doi.org/10.1177/0971890717 700533 


\section{Apéndice A}

Listado de factores de éxito y barreras

\section{Apéndice B}

Prueba $t$ de diferencia de medias

\begin{tabular}{|c|c|c|}
\hline Categoría & Factores de éxito (e) & Barreras en la promoción (b) \\
\hline \multirow{3}{*}{$\begin{array}{l}\text { Personales } \\
\text { cognitivo } \\
\text { emocionales }\end{array}$} & e01. Mi vocación y motivación & b04. Mi bajo nivel de autoestima \\
\hline & e02. Mi capacidad intelectual & b05. La falta de confianza en mi \\
\hline & acta, trabajo, dedicacion & $\begin{array}{l}\text { b06. La decisión personal de no mejorar } \\
\text { mi puesto académico (autorrestrición) }\end{array}$ \\
\hline \multirow{5}{*}{$\begin{array}{l}\text { Personales } \\
\text { familiares }\end{array}$} & e04. E1 apoyo emocional de mi familia & b01. Dificultad para conciliar mi vida \\
\hline & e05. E1 apoyo emocional de mi pareja & personal, familiar y laboral \\
\hline & $\begin{array}{l}\text { e06. La facilidad de conciliación de la vida } \\
\text { personal, familiar y laboral }\end{array}$ & $\begin{array}{l}\text { b02. E1 trabajo doméstico y de cuidados } \\
\text { b03. La falta de tiempo (asunción de }\end{array}$ \\
\hline & e07. La presencia de académicos en mi familia & una doble jornada: laboral y familiar) \\
\hline & $\begin{array}{l}\text { e08. Los factores socioeconómicos vinculados a } \\
\text { mi familia de origen }\end{array}$ & \\
\hline \multirow{4}{*}{$\begin{array}{l}\text { Organizacionales } \\
\text { grupales }\end{array}$} & $\begin{array}{l}\text { e09. "Estar en el momento justo en el lugar } \\
\text { adecuado" }\end{array}$ & $\begin{array}{l}\text { b07. E1 trato desigual por parte de } \\
\text { compañeros/as }\end{array}$ \\
\hline & $\begin{array}{l}\text { e10. La pertenencia a grupos de investigación } \\
\text { e11. Mentoring: apoyo del director/a de tesis, }\end{array}$ & $\begin{array}{l}\text { b08. La existencia de redes informales } \\
\text { de poder }\end{array}$ \\
\hline & jefe/a de departamento, etc. & b10. La falta de una red de apoyo \\
\hline & $\begin{array}{l}\text { e12. La posibilidad de realizar publicaciones } \\
\text { colectivas }\end{array}$ & (familiar, social, colegas, etc.) \\
\hline \multirow{4}{*}{$\begin{array}{l}\text { Organizacionales } \\
\text { profesionales }\end{array}$} & e13. La realización de estancias en el extranjero & b9. La diferenciación en la evaluación \\
\hline & $\begin{array}{l}\text { e14. La existencia de financiación externa } \\
\text { (convocatorias de investigación: proyectos, } \\
\text { ayudas para estancias, contratación personal } \\
\text { ayudante/formación, etc.) }\end{array}$ & $\begin{array}{l}\text { de méritos en base al género } \\
\text { b11. La falta de financiación y medios } \\
\text { económicos }\end{array}$ \\
\hline & $\begin{array}{l}\text { e15. La existencia de un sistema de habilitación } \\
\text { objetivo, justo y basado en el mérito (ASN) }\end{array}$ & \\
\hline & $\begin{array}{l}\text { e16. La existencia de un sistema de concurso } \\
\text { (promoción) objetivo, justo y basado en el mérito }\end{array}$ & \\
\hline \multirow{3}{*}{ Sociales } & & $\begin{array}{l}\text { b12. La existencia de una cultura } \\
\text { machista en la universidad }\end{array}$ \\
\hline & & $\begin{array}{l}\text { b13. La persistencia de estereotipos y } \\
\text { roles de género }\end{array}$ \\
\hline & & $\begin{array}{l}\text { b14. La persistencia de una sociedad } \\
\text { patriarcal }\end{array}$ \\
\hline
\end{tabular}

\section{Tablas de correlaciones Bivariadas. \\ Coeficiente Pearson}

\section{Apéndice $\mathrm{C}$}




\section{Apéndice D}

\section{Resumen de modelos de correspondencia múltiples}

\begin{tabular}{|c|c|c|c|c|c|c|c|c|c|c|}
\hline \multirow{2}{*}{\begin{tabular}{c}
\multicolumn{2}{c}{ Estadísticos } \\
Alfa de Cronbach
\end{tabular}} & \multicolumn{2}{|c|}{ Modelo 1} & \multicolumn{2}{|c|}{ Modelo 2} & \multicolumn{2}{|c|}{ Modelo 3} & \multicolumn{2}{|c|}{ Modelo 4} & \multicolumn{2}{|c|}{ Modelo 5} \\
\hline & & & & & & & & & & \\
\hline Dim.1 & & 0.624 & & 0.706 & & 0.699 & & 0.649 & & 0.729 \\
\hline Dim. 2 & & 0.6 & & 0.515 & & 0.535 & & 0.47 & & 0.579 \\
\hline Media & & 0.612 & & 0.628 & & 0.631 & & 0.574 & & 0.666 \\
\hline \multicolumn{11}{|l|}{ Inercia } \\
\hline Dim.1 & & 0.275 & & 0.298 & & 0.249 & & 0.29 & & 0.345 \\
\hline Dim.2 & & 0.263 & & 0.205 & & 0.177 & & 0.212 & & 0.254 \\
\hline Total & & 0.539 & & 0.503 & & 0.427 & & 0.502 & & 0.599 \\
\hline \multicolumn{11}{|l|}{$\%$ Varianza } \\
\hline Dim.1 & & 27.541 & & 29.81 & & 24.944 & & 28.954 & & 34.5 \\
\hline Dim.2 & & 26.318 & & 20.505 & & 17.709 & & 21.23 & & 25.356 \\
\hline Media & & 26.929 & & 25.158 & & 21.327 & & 25.092 & & 29.928 \\
\hline Medidas discriminantes & Dim.1 & Dim.2 & Dim.1 & Dim.2 & Dim.1 & Dim. 2 & Dim.1 & Dim.2 & Dim.1 & Dim.2 \\
\hline Sexo & 0.031 & 0.019 & 0.18 & 0.023 & 0.024 & 0.045 & 0.014 & 0.006 & 0.331 & 0.001 \\
\hline Categoría académica & 0.134 & 0.02 & 0.155 & 0.089 & 0.151 & 0.036 & 0.01 & 0.019 & 0.034 & 0.054 \\
\hline \multicolumn{11}{|l|}{ Factores de éxito } \\
\hline Vocación & 0.398 & 0.332 & & & & & & & & \\
\hline Capacidad intelectual & 0.12 & 0.33 & & & & & & & & \\
\hline Perseverancia & 0.159 & 0.31 & & & & & & & & \\
\hline Apoyo familiar & & & 0.174 & 0.591 & & & & & & \\
\hline Apoyo pareja & & & 0.248 & 0.641 & & & & & & \\
\hline Buena concili & & & 0.207 & 0.235 & & & & & & \\
\hline Estancias extranjero & & & & & 0.281 & 0.098 & & & & \\
\hline Financiación externa & & & & & 0.282 & 0.065 & & & & \\
\hline Sistema habilitación & & & & & 0.345 & 0.213 & & & & \\
\hline Sistema promoción & & & & & 0.368 & 0.259 & & & & \\
\hline Mentoring & & & & & 0.075 & 0.181 & 0.424 & 0.43 & & \\
\hline Publicación colectiva & & & & & 0.264 & 0.193 & 0.353 & 0.291 & & \\
\hline "Momento justo" & & & & & & & 0.434 & 0.245 & & \\
\hline Grupo investigación & & & & & & & 0.54 & 0.333 & & \\
\hline Familiares académicos & & & & & & & & & 0.002 & 0.038 \\
\hline Origen socioeconómico & & & & & & & & & 0.002 & 0.041 \\
\hline \multicolumn{11}{|l|}{ Barreras a la promoción } \\
\hline Baja autoestima & 0.625 & 0.45 & & & & & & & & \\
\hline Falta confianza & 0.624 & 0.354 & & & & & & & & \\
\hline Autorrestrición & 0.111 & 0.291 & & & & & & & & \\
\hline Valoración méritos & & & 0.437 & 0.122 & & & & & & \\
\hline Falta red de apoyo & & & 0.331 & 0.016 & & & & & & \\
\hline Trato de los pares & & & 0.455 & 0.042 & 0.387 & 0.314 & & & & \\
\hline Redes de poder & & & 0.495 & 0.086 & 0.416 & 0.337 & & & & \\
\hline Falta de financiación & & & & & 0.152 & 0.206 & & & & \\
\hline Trabajo doméstico & & & & & & & 0.208 & 0.12 & & \\
\hline Falta de tiempo & & & & & & & 0.333 & 0.255 & & \\
\hline Mala conciliación & & & & & & & & & 0.087 & 0.029 \\
\hline Cultura machista & & & & & & & & & 0.851 & 0.651 \\
\hline Estereotipos género & & & & & & & & & 0.769 & 0.66 \\
\hline Sociedad patriarcal & & & & & & & & & 0.684 & 0.555 \\
\hline Total activo & 2.203 & 2.105 & 2.683 & 1.845 & 2.744 & 1.948 & 2.316 & 1.698 & 2.76 & 2.028 \\
\hline$\%$ de varianza & 27.541 & 26.318 & 29.81 & 20.505 & 24.944 & 17.709 & 28.954 & 21.23 & 34.5 & 25.356 \\
\hline
\end{tabular}

Dim.1: Dimensión 1, Dim.2: Dimensión 2.
3 En las figuras correspondientes a los cinco modelos, los factores de éxito están representados con marcadores circulares y las barreras con marcadores de asterisco.

\section{Notas}

* Artículo de investigación. Este trabajo fue financiado por el Ministerio de Educación de España (ayuda FPU015/02850).

1 Edad y categoría académica tienen el mismo comportamiento debido a su relación (la promoción está vinculada a los años). Considerando el objetivo del estudio y la menor información que aporta la primera con respecto a la segunda, se utilizó la categoría académica.

2 Baja: valoraciones originales de 1 a 3 . Media: valoraciones originales de 4. Alta: valoraciones originales de 5 . 